\title{
CORRELATION BETWEEN CLINICAL AND ECHOCARDIOGRAPHIC FINDINGS WITH THE OCCURRENCE OF VENTRICULAR ARRHYTHMIAS IN PATIENTS WITH MITRAL VALVE PROLAPSE
}

\author{
Rasha Mohamed Abayazeed, Aly Abo-Elhoda , Mohamed Ayman Abdel-Hay, Sahar Azab, \\ Cardiology Department, Faculty of Medicine, Alexandria University, Alexandria, Egypt
}

\section{ABSTRAct}

Background: Chronic Mitral valve prolapse (MVP) has been linked to the occurrence of serious ventricular arrhythmias and sudden cardiac death. However, their mechanism is not fully understood particularly in the absence of haemodynamically significant mitral regurgitation (MR).

Objectives: The aim of the study is to identify if there are specific clinical and echocardiographic criteria that can predict the occurrence of ventricular arrhythmias in patients with mitral valve prolapse.

Methods:We prospectively enrolled 30 consecutive patients with MVP. Clinical examination, echocardiography (standard and speckle tracking) and 24 hours Holter monitoring were performed in all patients. The patients were further divided into 2 groups: arrhythmic MVP (7 patients) and non- arrhythmic MVP (23 patients) based on the presence of complex ventricular arrhythmias on Holter monitoring.

Results: Patients with arrhythmic MVP experienced syncope more frequently compared to the non- arrhythmia group ( 57 vs $4 \%, \mathrm{p}=0.006$ ) They also had larger left ventricular (LV) end systolic volume ( $45 \pm 8.8$ vs $36 \pm 8 \mathrm{ml}, \mathrm{p}=0.023$ ) despite similar LV ejection fraction (EF) and global longitudinal strain (GLS). The arrhythmic MVP patients had wider mitral annular disjunction $(7.7 \pm 3.8$ vs $3.9 \pm 3.9 \mathrm{~mm}, \mathrm{p}=0.033)$. There were no significant difference between the 2 groups regarding the degree of mitral regurgitation, leaflet affection (single or bileaflet) or mitral annular diameters.

Conclusion:Increased left ventricular end systolic volume and the degree of annular disjunction by echocardiography may contribute to the arrhythmic risk in patients with MVP irrespective of the presence and severity of mitral regurgitation.

Keywords: Mitral valve prolapse; ventricular arrhythmias; mitral annular disjunction

\section{INTRODUCTION}

Mitral valve prolapse (MVP) is a common cardiac valvular abnormality affecting about $2-3 \%$ of the general population. ${ }^{(1-4)}$ It is defined as systolic displacement of one or both mitral valve leaflets $>2 \mathrm{~mm}$ into the left atrium, with or without leaflet thickening, beyond the long axis of mitral annulur plane e $^{(4,5)}$
MVP is not a completely understood clinical entity, despite being known for more than a century. Although it is generally considered as a benign condition, ${ }^{(1,3)}$ it has been associated with various clinical complications including significant mitral regurgitation, congestive heart failure, infective endocarditis and stroke; even ventricular arrhythmias and sudden cardiac death have been reported. ${ }^{(8-10)}$

The mechanism of ventricular arrhythmias and sudden cardiac death in patients with MVP is not fully understood particularly in the absence of hemodynamically significant mitral regurgitation. Several theories were suggested to explain the mechanism of significant ventricular arrhythmias in MVP patients but most of them are still speculative. These include MVPrelated factors such as the excessive traction on the papillary muscles (PMs) by the prolapsing leaflets; ${ }^{(12)}$ the mechanical stimulation of the endocardium by the elongated chordae, with after depolarization induced triggered activity; the diastolic depolarization of muscle fibers in redundant leaflets with triggered repetitive automaticity; (13) and the endocardial friction lesions with the extension into the myocardium. ${ }^{(14)}$ Myocardial substrate of the electrical instability in patients with MVP has been addressed and LV fibrosis was detected in histology at the level of PMs as well as in the LV infero-basal wall $^{(15)}$

Till now, there are no definite criteria that can predict the incidence of life threatening ventricular arrhythmias in patients with MVP. We thought that by combining clinical, electrocardiographic and echocardiographic findings in patients with MVP, we may be able to provide more specific criteria to identify patients with MVP 
liable to suffer from serious ventricular arrhythmias.

\section{METHODS:}

\section{Study population:}

In this prospective cross-sectional study, 30 MVP patients were consecutively recruited from our adult echocardiography lab after approval of the Institutional Ethics Committee. MVP was defined as systolic displacement of one or both mitral valve leaflets $>2 \mathrm{~mm}$ into the left atrium beyond the plane of the mitral annulus in the parasternal long axis view on transthoracic echocardiography. ${ }^{(4)}$ Exclusion criteria were Patients with possible alternative cause for arrhythmia (e.g: coronary artery disease, long or short QT syndromes, Brugada syndrome, arrhythmogenic cardiomyopathy, hypertrophic cardiomyopathy and electrolyte disturbances) and patients with left bundle branch block (LBBB), conduction delay or paced rhythm.

All patients underwent history taking, clinical examination, echocardiography and $24 \mathrm{~h}$ Holter monitoring,

Study cases were further divided into arrhythmic MVP (A-MVP) and non arrhythmic MVP (NA-MVP) groups based on the presence of complex ventricular arrhythmias on 24 hours Holter monitoring. Complex ventricular arrhythmia was defined as the presence of couplets, sustained or non-sustained ventricular tachycardia (VT) and $\mathrm{R}$ on $\mathrm{T}$ pattern ventricular ectopy.

All participants gave written informed consent.

\section{Echocardiography:}

The echocardiographic studies were performed iE 33 machine (Philips Medical Systems, Andover, MA, USA). Mitral valve morphology and function were assessed including annular diameters, leaflet thickness, degree of displacement, presence and degree of annular disjunction and severity of mitral regurgitation. Ejection fraction (EF) was calculated by modified Simpson's biplane method.(16) Atrial diameter was determined by M-mode or $2 \mathrm{D}$ echocardiography in the parasternal long-axis plane and left atrial volume was calculated using the area length method corrected for body surface area [left atrial volume index (LAVI)]. ${ }^{(16)}$

\section{Speckle tracking echocardiography (STE):}

Longitudinal strain was obtained from three apical views at frame rate $>50 / \mathrm{s}$. Segments that failed to track were manually adjusted. Region of interest (ROI) was adjusted to fit the average of the myocardial thickness. LV GLS was defined as the average of peak longitudinal strains from a17 LV segments model. ${ }^{(17)}$

\section{Holter monitoring:}

All patients underwent 24 hours three channel Holter monitoring to detect the presence and frequency of ventricular premature contractions (VPCs).

\section{STATISTICAL ANALYSIS:}

Data were fed to the computer and analyzed using IBM SPSS software package version 20.0. (Armonk, NY: IBM Corp). Qualitative data were described using number and percent Quantitative data were described using range (minimum and maximum), mean and standard deviation. The Kolmogorov-Smirnov test was used to verify the normality of distribution. Significance of the obtained results was judged at the 5\% level. The used tests were: Chi-square test, Fisher's Exact or Monte Carlo correction as correction for chi-square; and Student t-test.

\section{RESULTS:}

\section{Comparison between the A-MVP and NA- MVP patients:}

\section{Basic clinical characteristics:}

A-MVP patients experienced syncope more frequently than the NA-MVP group. They also had greater utilization of beta- blockers (BB). No other significant differences between the 2 groups regarding baseline characters or resting ECG findings.

Basic clinical characteristics of the 2 groups are summarized in table (1).

\section{Echocardiographic findings:}

The A-MVP cases had larger LV end systolic volume (ESV) compared to the NA-MVP group $(\mathrm{p}=0.023)$ despite similar LVEF and GLS and similar grades of MR. They also had wider mitral annular disjunction $(\mathrm{p}=0.033$, figures 1). There was no significant difference in other echocardiographic parameters between the 2 groups. Echocardiographic characteristics of the 2 groups are summarized in table ${ }^{(2)}$

\section{Holter monitoring:}

Seven patients had complex ventricular arrhythmias (figure 2). Non sustained ventricular tachycardia (NSVT) was the most frequent form (3 patients, $43 \%$ ) followed by couplets (2 patients, 28.6\%). One patient had NSVT and ventricular premature contraction (VPC) burden of $18 \%$ /day and one patient had Ron $\mathrm{T}$ pattern ventricular ectopy and VPC burden of 17\%/day 
Table (1): Clinical characteristics of mitral valve prolapse patients with and without ventricular arrhythmia:

\begin{tabular}{|c|c|c|c|}
\hline Clinical characteristics & $A-M V P(N=7)$ & NA-MVP $(n=23)$ & P value \\
\hline Age, years mean (SD) & $39(15)$ & $29(11)$ & 0.068 \\
\hline Female sex.n(\%) & $4(57)$ & $22(95.7)$ & 0.031 \\
\hline BSA,m2 mean(SD) & $1.8(0.2)$ & $1.7(0.1)$ & 0.080 \\
\hline $\mathrm{HR}, \mathrm{bpm}$ mean (SD) & $70(11)$ & $80(12)$ & 0.087 \\
\hline Svstolic BP. mmHg mean(SD) & $115.8(12)$ & $112.6(10)$ & 0.507 \\
\hline Diastolic BP, mmHg mean (SD) & $77.50(10.55)$ & $73.42(7.45)$ & 0.535 \\
\hline UTN n $(0 / 2)$ & $1(142)$ & $1(132)$ & $n \Lambda 18$ \\
\hline $\mathrm{DM}, \mathrm{n}(\%)$ & 0 & 0 & \\
\hline CAD.n $(\%)$ & 0 & 0 & \\
\hline Tobacco use, n (\%) & 0 & $1(4.3)$ & 1.000 \\
\hline Palpitations, n (\%) & $7(100)$ & $19(82.6)$ & 0.548 \\
\hline Chest pain, n (\%) & $3(43)$ & $13(56.5)$ & 0.325 \\
\hline Syncope, n (\%) & $4(57)$ & $1(4.3)$ & $0.006^{*}$ \\
\hline Beta-blockers. n (\%) & $3(43)$ & $1(4.3)$ & $0.031^{*}$ \\
\hline
\end{tabular}

BSA, body surface area; CAD, coronary artery disease; DM, diabetes mellitus; HR, heart rate; HTN, hypertension.

*: Statistically significant at $\mathrm{p} \leq 0.05$

Table (2): Echocardiographic characteristics of mitral valve prolapse patients with and without ventricular arrhythmia

\begin{tabular}{|c|c|c|c|}
\hline Echocardiographic characteristics & $\operatorname{A-MVP}(n=7)$ & NA-MVP $(n=23)$ & $P$ value \\
\hline LAVI, ml/m2 mean(SD) & $29(6)$ & $31.76(13.55)$ & 0.169 \\
\hline Annular disjunction, $\mathrm{n}(\%)$ & $6(85.7 \%)$ & $13(56.5 \%)$ & 0.215 \\
\hline Annular disiunction. mm mean (SD) & $7.7(3.8)$ & $3.9(3.9)$ & $0.033^{*}$ \\
\hline LVEDV, ml mean (SD) & $110.1(27)$ & $97.4(20.5)$ & 0.191 \\
\hline LVESV, ml mean(SD) & $45(8.8)$ & $36.3(8.2)$ & $0.023^{*}$ \\
\hline LVEF, \% mean (SD) & $59(3.2)$ & $62.3(4.2)$ & 0.064 \\
\hline GLS, $\%$ mean (SD) & $-21.6(-1.7)$ & $-22.5(-2.4)$ & 0.358 \\
\hline A-P annular diameter, mm mean (SD) & $34.9(4.6)$ & $34(4.9)$ & 0.680 \\
\hline Bicommissural annular diameter, mm mean (SD) & $36.9(6.2)$ & $36.3(5.9)$ & 0.832 \\
\hline Bileaflet prolapse, $\mathrm{n}(\%)$ & $6(85.7 \%)$ & $18(78.3 \%)$ & 1.000 \\
\hline No significant MR, n (\%) & $3(42.9 \%)$ & $10(43.5 \%)$ & \multirow{4}{*}{0.132} \\
\hline Mild MR, n (\%) & $4(57.1 \%)$ & $4(17.4 \%)$ & \\
\hline Moderate MR, n (\%) & 0 & $5(21.7 \%)$ & \\
\hline Severe MR, n (\%) & 0 & $4(17.4 \%)$ & \\
\hline
\end{tabular}

GLS: global longitudinal strain; LAVI, left atrial volume index; LV, left ventricle; LVEDV, left ventricular end diastolic volume;

LVEF, left ventricular ejection fraction; LVESV, left ventricular end systolic volume; MR, mitral regurgitation.

*: Statistically significant at $\mathrm{p} \leq 0.05$ 


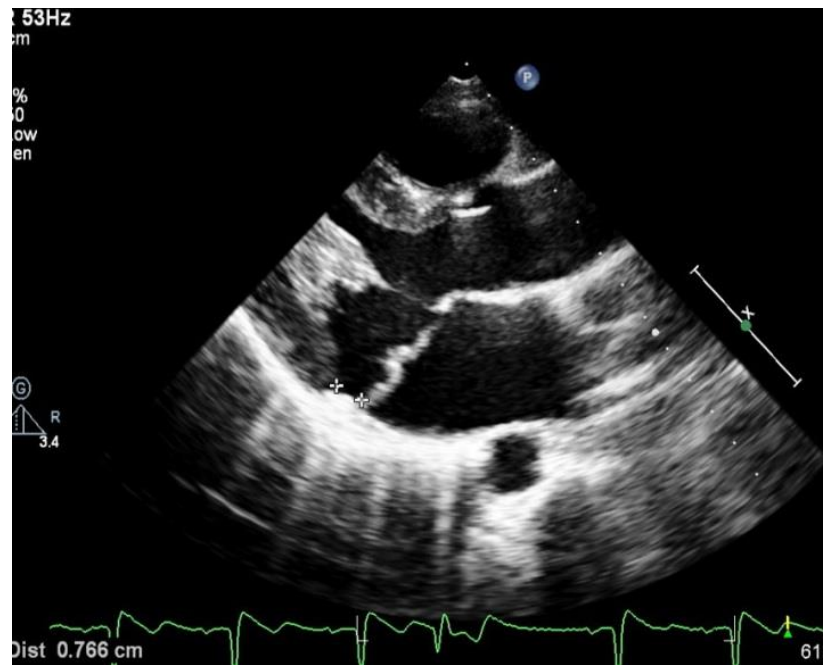

Figure (1): Mitral annular disjunction in patient with bileaflet prolapse

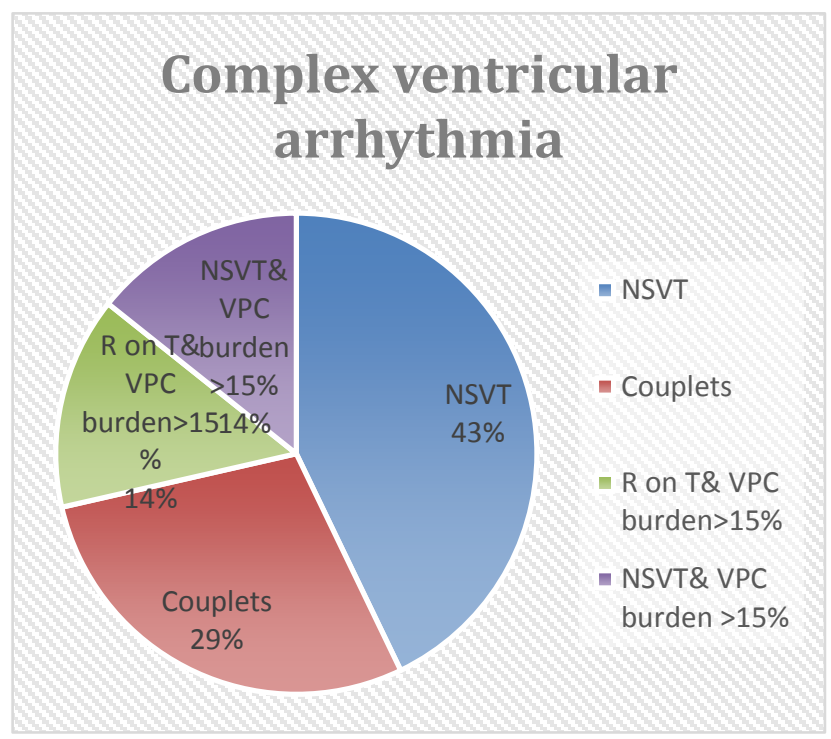

Figure (2): Distribution of ventricular arrhythmias in the arrhythmic MVP group

\section{DISCUSSION:}

In our study, complex ventricular arrhythmias was found in $23 \%$ of the study population. Although more than $40 \%$ of the patients in the arrhythmia group was maintained on BBs, this failed to control the ventricular arrhythmia and one patient on BB therapy had VPC burden of 17\%/day. This finding is disappointing but not surprising. In a study done by Basso et al on sudden cardiac death (SCD) and MVP, ${ }^{(15)} 21 \%$ of young adult SCD victims and two living patients had aborted SCD despite beta-blocker therapy.The ability of BB to control ventricular arrhythmias in the setting of MVP is questionable and its role is less established than in many other diseases.

There was no significant difference in the number of patients having annular disjunction in both groups, but the degree of annular disjunction was significantly larger in the arrhythmia group in agreement with several previous studies. ${ }^{(18-20)}$ There is an increasing interest in the association between mitral annular disjunction (MAD) and ventricular arrhythmias and a recent study had reported that MAD itself is arrhythmogenic even in the absence of MVP. ${ }^{(19)} \quad$ Unlike previous studies, bileaflet prolapse was not a predictor of ventricular arrhythmia in our study. ${ }^{(10,15,20)}$ Although a previous study ${ }^{(21)}$ found that the presence of moderate to severe mitral regurgitation is the only independent predictor for occurrence of arrhythmia in MVP, several subsequent studies showed that ventricular arrhythmia can occur in MVP in the absence of significant MR. ${ }^{(15,20)}$ In our study population, all patients in the arrhythmia group had no or mild MR asserting the belief that MVP itself is arrhythmogenic even in the absence of hemodynamically significant MR. The increased LVESV in the arrhythmia group supports the theory of myocardial substrate being a possible mechanism of ventricular arrhythmia in MVP. ${ }^{(15)}$ No significant difference in EF and GLS was found between the 2 groups and the same observation was reported in a recent study on arrhythmic MVP. ${ }^{(20)}$

Although female sex was significantly higher among the NA- MVP patients, this is most probably a selection bias as $87 \%$ of the total study population were females.

\section{STUDY LIMITATIONS:}

Small number of patients included in the study.

\section{CONCLUSIONS:}

Increased left ventricular end systolic volume and the degree of annular disjunction by echocardiography may contribute to the arrhythmic risk in patients with MVP irrespective of the presence and severity of mitral regurgitation.

\section{REFERENCES:}

1. Freed LALevy D, Levine RA, Larson MG, Evans JC, Fuller DL, et al. Prevalence and clinical outcome of mitral-valve prolapse. N Engl J Med 1999;341:1-7.

2. Devereux RB, Jones EC, Roman MJ, Howard BV, Fabsitz RR, Liu JE, et al. Prevalence and correlates of mitral valve prolapse in a population-based sample of American Indians: the Strong Heart Study. Am J Med2001;111:679-85. 
3. Freed LA, Benjamin EJ, Levine RA, Larson MG, Evans JC, Fuller DL, et al. Mitral valve prolapse in the general population: the benign nature of echocardiographic features in the Framingham heart study. J Am Cardiol 2002;40:1298-304.

4. Delling FN, Vasan RS. Epidemiology and pathophysiology of mitral valve prolapse: new insights into disease progression, genetics, and molecular basis. Circulation 2014; 129:2158-70.

5. Levine RA, Stathogiannis A, Newell JB, Harrigan B, Weyman AE. Reconsideration of echocardiographic standards for mitral valve prolapse: lack of association between leaflet displacement isolated to the apical four chamber view and independent echocardiographic evidence of abnormality. J Am Coll Cardiol 1988;11: 1010-9

6. Devereux RB, Brown WT, Kramer-Fox R, Sachs I Inheritance of mitral valve prolapse: effect of age and sex on gene expression. Ann Intern Med 1982;97:826-32.

7. Strahan MV, Murphy EA, Fortuin NJ, Come PC, Humphries JO. Inheritance of the mitral valve prolapse syndrome: discussion of a three dimensional penetrance model. Am J Med 1983;74:967-72.

8. Marks AR, Choong CY, Snfilippo AJ, Weyman AE. Identification of high-risk and low-risk subgroups of patients with mitral-valve prolapse. N Engl J Med 1989; 320: 1031-6.

9. Avierinos JF, Gersh BJ, Melton LJ III, Bailey KR, Shub C, Nishimura RA, et al. Natural history of asymptomatic mitral valve prolapse in the community. Circulation 2002;106:1355-61.

10. Sriram CS, Syed FF, Ferguson ME, Johnson JN, Enriquez-Sarano M, Cetta F, et al. Malignant bileaflet mitral valve prolapse syndrome in patients with otherwise idiopathic out-of-hospital cardiac arrest. J Am Coll Cardiol 2013;62:222-30.

11. Lichstein E. Site of origin of ventricular premature beats in patients with mitral valve prolapse. Am Heart J 1980;100:450-7.

12. Cobbs BW Jr, King SB $3^{\text {rd }}$. Ventricular buckling: a factor in the abnormal ventriculogram and peculiar hemodynamics associated with mitral valve prolapse. Am Heart J 1977;93:741-58.
13. Salazar AE, Edwards JE. Friction lesions of ventricular endocardium. Relation to chordae tendineae of mitral valve. Arch Pathol 1970;90:364-79.

14. Barlow JB, Bosman CK. Aneurysmal protrusion of the posterior leaflet of the mitral valve. An auscultatoryelectrocardiographic syndrome. Am Heart J 1966; 71:166-78.

15. Basso C, Perazzolo Marra M, Rizzo S, De Lazzari M, Giorgi B, Cipriani A, et al. Arrhythmic mitral valve prolapse and sudden cardiac death. Circulation 2015; 132:556-66.

16. Lang RM, Badano LP, Mor- Avi V, Afilalo J, Armstrong A, Ernande L, et al. Recommendations for Cardiac Chamber Quantification by Echocardiography in Adults: An Update from the American Society of Echocardiography and the European Association of Cardiovascular Imaging. J Am Soc Echocardiogr 2015; 28:1-39.

17. Mor-Avi V, Lang RM, Badano LP, Belohlavec M, Cardim NM, Derumeaux G, et al. Current and evolving echocardiographic techniques for the quantitative evaluation of cardiac mechanics: ASE/EAE consensus statement on methodology and indications endorsed by the Japanese Society of Echocardiography. J Am Soc Echocardiogr 2011;24:277-313.

18. Carmo P, Andrade MJ, Aguiar C, Rodrigues R, Gouveia $r$, Silva JA. Mitral annular disjunction in myxomatous mitral valve disease: a relevant abnormality recognizable by transthoracic echocardiography. Cardiovasc Ultrasound 2010; 8:53.

19. Dejgaard LA, Skjølsvik ET Lie ØH, Ribe M, Stokke MK, Hegbom F, et al. The Mitral Annulus Disjunction Arrhythmic Syndrome. J Am Coll Cardiol 2018; 72: 1600-9.

20. Ermakov S, Gulhar R, Lim L, Bibby D, Fang Q, Nah G, et al. Left ventricular mechanical dispersion predicts arrhythmic risk in mitral valve prolapse. Heart 2019; 106;163-9.

21. Turker Y, Ozaydin M, Acar G, Ozgul M, Hoscan Y, Varol E, et al. Predictors of ventricular arrhythmias in patients with mitral valve prolapse. Int $\mathrm{J}$ Cardiovasc Imaging 2010;26:139-45 